\title{
Study on the Effects of Different Dietary Preparation Methods on Capsule Endoscopy
}

\author{
Shizhen Zhang1, Jingping Cheng ${ }^{2 *}$ \\ ${ }^{1}$ School of Medicine, Wuhan University of Science and Technology, Wuhan, China \\ ${ }^{2}$ China Resources \& WISCO General Hospital, Wuhan University of Science and Technology, Wuhan, China \\ Email: *404178516@qq.com
}

How to cite this paper: Zhang, S.Z. and Cheng, J.P. (2020) Study on the Effects of Different Dietary Preparation Methods on Capsule Endoscopy. International Journal of Clinical Medicine, 11, 236-241. https://doi.org/10.4236/ijcm.2020.115024

Received: March 23, 2020

Accepted: May 10, 2020

Published: May 13, 2020

Copyright (c) 2020 by author(s) and Scientific Research Publishing Inc. This work is licensed under the Creative Commons Attribution International License (CC BY 4.0).

http://creativecommons.org/licenses/by/4.0/

\begin{abstract}
Background: Capsule endoscopy (CE) examination has become the first-line diagnostic method for small bowel disease, and intestinal preparation is a key factor affecting the quality of $\mathrm{CE}$ examination. At present, there is no uniform standard for dietary preparation methods before $\mathrm{CE}$ at home and abroad, and there are few systematic comparative studies on the choice of dietary methods. Objective: To explore the best method of preparing diet for capsule endoscopy (CE) examination. Methods: 93 Patients who underwent CE examination in the digestive endoscopy center of Wuhan union medical college hospital in October 2019 were randomly divided into 3 groups. Group A ( $\mathrm{n}=$ 31): 1 day before the examination; a diet with low-residue was given, and 1 day before the examination, fasting was started at 8 p.m. until the examination was completed. Group B $(\mathrm{n}=30)$ : a clear liquid diet was followed 1 day before the examination, and fasting was started at 8 p.m. 1 day before the examination until the examination was completed. Group C $(\mathrm{n}=32)$ : followed an ordinary diet 1 day before the examination, and began fasting at 8 p.m. 1 day before the examination until the examination was completed. Intestinal preparation of the same drug was performed in 3 groups, and the incidence of side effects and intestinal cleanliness of each group were compared. Results: There was no significant difference in cleanliness between the three groups $(\mathrm{P}>0.05)$. The incidence of side effects in the three groups was $28.13 \%, 70.00 \%$ and $21.88 \%$, respectively. The difference between group A and group $B$, group $C$ and group $B$ is statistically significant $(P<0.05)$. Conclusion: The three diet preparation methods of low-residue diet, clear liquid diet and ordinary diet can all achieve good intestinal cleansing effect. Clear liquid diet can not obviously improve the cleanliness of the small intestine, and there are relatively many side effects.
\end{abstract}

${ }^{\star}$ Corresponding author. 


\section{Keywords}

Capsule Endoscopy, Intestinal Preparation, Diet

\section{Introduction}

Capsule endoscopy (CE) examination has the advantages of no pain, simple operation, safety, non-invasiveness, and no cross infection, which greatly expands the field of vision of digestive tract examination. With the rapid development of gastrointestinal endoscopy technology, small intestine capsule endoscopy has become the preferred method for diagnosing small intestinal diseases in the field of digestive diseases. However, there are still some shortcomings in the endoscopy of small intestine capsules. Intestinal contents play a decisive role in the accuracy of diagnosis.

Intestinal preparation is a key element that affects the quality of CE examination [1] [2] [3]. If the gastrointestinal tract is not cleaned thoroughly, and the contents of the intestinal tract remain, it may result in unsatisfactory captured images, which may affect the examination results and even cause misdiagnosis and missed diagnosis. Therefore, thorough gastrointestinal cleansing before capsule endoscopy is performed to improve the clarity of the captured images, which is helpful to improve the accuracy of small bowel disease detection.

Dietary preparation is an important auxiliary measure for intestinal preparation [4] [5]. According to the amount of residue generated, dietary preparation can be divided into clear diet, less residue diet and ordinary diet. At present, there is no uniform standard for dietary preparation methods before $\mathrm{CE}$ at home and abroad, and there are few systematic comparative studies on the choice of dietary methods. In order to find out the best diet preparation methods before CE examination, this article compares and studies the differences in the effect of different diet preparation protocols on capsule endoscopic bowel preparation.

\section{Materials and Methods}

\subsection{General Information}

The case data were included in 93 patients who underwent capsule endoscopy in the digestive endoscopy center of wuhan union medical college hospital in October 2019, including 60 males and 33 females aged 15 - 89 years. Exclusion criteria: (1) failure of total intestinal examination caused by device factor; (2) the capsule lens did not reach the small intestine and the capsule lens did not complete the examination; (3) gastrointestinal bleeding, eating in advance and other factors interfere with the examination of small intestine cleanliness.

\subsection{Methods of Examination}

(1) Inform the subjects and their families of the precautions for CE examination, 
and sign the informed consent. (2) Preparation of intestinal tract: Before the examination, group A $(n=31)$, group $B(n=30)$ and group $C(n=32)$ were given a slag-free diet, a clear-liquid diet and a normal diet, and the rest were the same. That is to say, fasting will be started at 8:00 p.m. before the inspection until the inspection is completed; at 9:00 p.m. before the inspection on the 1st before inspection, one packet of compound polyethylene glycol (PEG, Fujingqing) will be dissolved in the morning at 5:00 and 6:00 on the inspection day. Put it in $1 \mathrm{~L}$ of warm water and finish it in 1 hour. On the day of the inspection at 7:00, a bottle of simethicone is dissolved in $200 \mathrm{ml}$ of cold water and finished in $5 \mathrm{mi}$ nutes. (3) Inspection: After swallowing CE with a small amount of water, the subjects are instructed to fast and not to stay away from the inspection site. The doctor monitors the operation of the capsules in real time and informs them to eat according to the monitoring results.

\subsection{Evaluation Method}

Observe the cleanliness of the small intestine, calculatethe incidence of side effects. Small intestine cleanliness is evaluated by a single-blind method, which is scored by $\mathrm{CE}$ readers based on the cleanliness of the intestinal tract of the $\mathrm{CE}$ image and the overall observation effect. Intestinal cleaning grading: Level 1: there are very few bubbles in the small intestine, no residue and turbid liquid. The observation effect is good; Level 2: there is a small amount of air bubbles or turbid liquid in the small intestine, no residue, which has little impact on the observation effect; Level 3: a large amount of air bubbles or turbid liquid is found in the small intestine, and a little residue, which affects the observation effect; Level 4: a large amount bubbles and turbid liquid, more residue gathered in the small intestine, which seriously affect the observation. Side effects include gastrointestinal symptoms such as abdominal pain, nausea, and vomiting, as well as general malaise such as dizziness and palpitation. The incidence of side reactions is the percentage of people with side reactions in the total number of people.

\subsection{Statistical Methods}

SPSS 22.0 statistical software was used for statistical analysis. The small intestine cleanliness was measured by the rank sum test, the measurement data were expressed by mean \pm standard deviation $(x \pm s)$, and the incidence of side reactions was measured by chi-square test of line and column list data, with the test standard $=0.05$.

\section{Results}

\subsection{Comparison of General Conditions of Patients}

General statistics of gender and age of patients in the three groups: there was no significant difference in gender and age among the three groups, and the groups were comparable (Table 1 ). 
Table 1. Comparison of general conditions of patients.

\begin{tabular}{|c|c|c|c|c|}
\hline \multirow{2}{*}{ Groups } & \multirow{2}{*}{$\mathrm{n}$} & \multicolumn{2}{|c|}{ Gender } & \multirow{2}{*}{ Age (years) } \\
\hline & & M & $\mathrm{F}$ & \\
\hline Group A & 31 & 18 & 13 & $42.77 \pm 15.34$ \\
\hline Group B & 30 & 21 & 9 & $43.77 \pm 12.47$ \\
\hline Group C & 32 & 21 & 11 & $43.00 \pm 13.24$ \\
\hline
\end{tabular}

\subsection{Comparison of the Three Groups Related Indicators}

There was no significant difference in cleanliness of small intestine between groups $\mathrm{A}, \mathrm{B}$ and $\mathrm{C}(\mathrm{P}>0.05)$. The results showed that during $\mathrm{CE}$ examination, taking a sufficient amount of laxatives, most can achieve good intestinal preparation, but the relationship with dieting seems not so close. Among the $37 \mathrm{pa}$ tients, 11 had abdominal pain and distension, 7 had nausea and vomiting, and 19 had palpitation, dizziness and chills. The incidences of side effects in groups A, B, and C were $28.13 \%, 70.00 \%$, and $21.88 \%$, respectively, with statistically significant differences between group $\mathrm{A}$ and $\mathrm{B}, \mathrm{C}$ and $\mathrm{B}(\mathrm{P}<0.05)$. The results showed that there were significant differences in side effects such as abdominal pain, nausea, or dizziness in the normal diet group and the low-residue diet group compared with the clear liquid diet group. See Table 2.

\section{Conclusion}

The three diet preparation methods of low-residue diet, clear liquid diet and ordinary diet can all achieve good intestinal cleansing effect. Clear liquid diet can not obviously improve the cleanliness of the small intestine, and there are relatively more side effects. The key part of intestinal preparation before capsule endoscopy is cleaning the intestines. Taking a sufficient amount of laxatives strictly according to doctor's instructions until clear liquid is excreted, most can achieve good intestinal preparation effects. And the relationship between cleanliness and dieting seems not so close. Furthermore, patients have fewer side effects after eating, and will be more tolerant to capsule endoscopy examination.

\section{Discussion}

Since the capsule endoscopy was introduced in 1999, the clinical application of capsule endoscopy has developed rapidly [6], and capsule endoscopy has now become the preferred diagnostic method for small bowel diseases [7]. However, due to the lack of water injection, gas injection, suction, and controllable functions of the capsule endoscope, the real-time intervention of the shooting field cannot be performed, so the $\mathrm{CE}$ examination has higher requirements for cleanliness of the small intestine [8] [9]. In addition to the quality of intestinal preparation and oral laxatives, it is closely related to diet preparation programs [10] [11]. There are few systematic comparisons of diet preparation methods before the CE examination. At present, it is generally believed that the effect of a clear liquid diet is better, but it is unclear whether the clear liquid diet before the test 
Table 2. Comparison of the three groups related indicators.

\begin{tabular}{cccccc}
\hline \multirow{2}{*}{ Groups } & Cleanliness of small intestine/case & Level 4 & $\begin{array}{c}\text { The incidence of side } \\
\text { reactions/case (\%) }\end{array}$ \\
\cline { 2 - 5 } & Level 1 & Level 2 & Level 3 & 8 & $9(29.03)$ \\
Group A $(\mathrm{n}=31)$ & 5 & 14 & 6 & 3 & $21(70.00)$ \\
Group B $(\mathrm{n}=30)$ & 7 & 14 & 10 & 3 & $7(21.88)$ \\
Group C $(\mathrm{n}=32)$ & 4 & 15 & & 2 & \\
\hline
\end{tabular}

has sufficient benefits to patients compared with low-residue diet and the normaldiet [12] [13] [14] [15].

Intestinal preparation is one of the key aspects of capsule endoscopy. At present, it mainly focuses on cleaning the intestines and preparing food. We use 3 L PEG electrolyte intestinal lavage liquid for intestinal preparation, and the effect is satisfactory [16]. As for diet preparation, some are accustomed to restricting their diet $1 \mathrm{~d}$ before the examination and fasting from 8:00 p.m. the day before the examination. Due to lack of energy and nutritional supplements, the tolerance of the testers is significantly decreased, especially in poor health or with basic diseases. The elderly, or outpatients from afar, are more likely to have hypoglycemia-like reactions, aggravate discomfort, and directly affect the examination results. We observed the intestinal cleanliness of the three groups with different dietary conditions, and the results were statistically analyzed, with $\mathrm{P}>$ 0.05 . There was no statistical difference in intestinal cleanliness among the three groups. The three diets had little effect on capsule endoscopy. A clear liquid diet cannot significantly improve the cleanliness of the small intestine, and there are relatively more side effects. There are still some deficiencies in this study, we need to expand the sample size and do more detailed researches. And it's necessary to do systematic comparative studies on the choice of dietary methods in the following time.

\section{Conflicts of Interest}

The authors declare no conflicts of interest regarding the publication of this paper.

\section{References}

[1] Shim, K.N., Jeon, S.R., Jang, H.J., et al. (2017) Quality Indicators for Small Bowel Capsule Endoscopy. Clinical Endoscopy, 50, 148-160. https://doi.org/10.5946/ce.2017.030

[2] Song, H.J., Moon, J.S., Do, J.H., et al. (2013) Guidelines for Bowel Preparation before Video Capsule Endoscopy. Clinical Endoscopy, 46, 147-154. https://doi.org/10.5946/ce.2013.46.2.147

[3] Kotwal, V.S., Attar, B.M., Gupta, S., et al. (2014) Should Bowel Preparation, Antifoaming Agents, or Prokinetics Be Used before Video Capsule Endoscopy? A Systematic Review and Meta-Analysis. European Journal of Gastroenterology \& Hepatology, 26, 137-145. https://doi.org/10.1097/MEG.0b013e328365b9d4

[4] Hassan, C., Bretthauer, M., Kaminski, M.F., et al. (2013) European Society of Ga- 
strointestinal Endoscopy. Bowel Preparationfor Colonoscopy: European Society of Gastrointestinal Endoscopy (ESGE) Guideline. Endoscopy, 45, 142-150. https://doi.org/10.1055/s-0032-1326186

[5] Mathus-Vliegen, E., Pellisé, M., Heresbach, D., et al. (2013) Consensus Guidelines for the Use of Bowel Preparation Priorto Colonic Diagnostic Procedures: Colonoscopy and Small Bowel Video Capsule Endoscopy. Current Medical Research and Opinion, 29, 931-945. https://doi.org/10.1185/03007995.2013.803055

[6] Connor A., Tolan D., Hughes S., et al. (2012) Consensus Guidelines for the Safe Prescription and Administration of Oral Bowel-Cleansing Agents. Gut, 61, 1525-1532. https://doi.org/10.1136/gutjnl-2011-300861

[7] Pennazio, M., Spada, C., Eliakim, R., et al. (2015) Small-Bowel Capsule Endoscopy and Device-Assisted Enteroscopy for Diagnosis and Treatment of Small-Bowel Disorders: European Society of Gastrointestinal Endoscopy (ESGE) Clinical Guideline. Endoscopy, 47, 352-376. https://doi.org/10.1055/s-0034-1391855

[8] Chen, H.L., Tang, J.G., Su, Y.Q., et al. (2017) Effect of Moxapride on the Quality of Capsule Endoscopy. Journal of Clinical Gastroenterology, 29, 301-303.

[9] Enns R A., Hookey L., Armstrong D., et al. (2017) Clinical Practice Guidelines for the Use of Video Capsule Endoscopy. Gastroenterology, 152, 497-514. https://doi.org/10.1053/j.gastro.2016.12.032

[10] Berry, M.A. and DiPalma, J.A. (1994) Orthograde Gut Lavage for Colonoscopy. Alimentary Pharmacology \& Therapeutics, 8, 391-395. https://doi.org/10.1111/j.1365-2036.1994.tb00305.x

[11] Saltzman, J.R., et al. (2015) ASGE Standards of Practice Committee. Bowel Preparation before Colonoscopy. Gastrointestinal Endoscopy, 81, 781-794.

[12] Seo, E.H., Kim, T.O., Park, M.J., et al. (2013) Low-Volume Morning-Only Polyethylene Glycol with Specially Designed Test Meals versus Standard-Volume Split-Dose Polyethylene Glycol with Standard Diet for Colonoscopy: A Prospective, Randomized Trial. Digestion, 88, 110-118. https://doi.org/10.1159/000353244

[13] Sipe, B.W., Fischer, M., Baluyut, A.R., et al. (2013) A Low-Residue Diet Improved Patient Satisfaction with Split-Dose Oral Sulfate Solution without Impairing Colonic Preparation. Gastrointestinal Endoscopy, 77, 932-936. https://doi.org/10.1016/j.gie.2013.01.046

[14] Soweid, A.M., Kobeissy, A.A., Jamali, F.R., et al. (2010) A Randomized Single-Blind Trial of Standard Diet versus Fiber-Free Diet with Polyethylene Glycol Electrolyte Solution for Colonoscopy Preparation. Endoscopy, 42, 633-638. https://doi.org/10.1055/s-0029-1244236

[15] Melicharkova, A., Flemming, J., Vanner, S., et al. (2013) A Low-Residue Breakfast Improves Patient Tolerance without Impacting Quality of Low-Volume Colon Cleansing Prior to Colonoscopy: A Randomized Trial. American Journal of Gastroenterology, 108, 1551-1555. https://doi.org/10.1038/ajg.2013.21

[16] Zhu, S.G., Liao, Z. and Li, Z.S. (2017) The Status and Progress of Capsule Endoscopy Bowel Preparation Protocol. Chinese Journal of Gastroenterology, 34, 519-522. 